\title{
BMJ Open Clinical characteristics of importance to outcome in patients with axial spondyloarthritis: protocol for a prospective descriptive and exploratory cohort study
}

Rikke Asmussen Andreasen, ${ }^{1,2}$ Lars Erik Kristensen, ${ }^{2}$ Torkell Ellingsen, ${ }^{3,4}$ Robin Christensen, ${ }^{2}$ Xenofon Baraliakos, ${ }^{5} \mathrm{Jimmi}$ Wied, ${ }^{6}$ Claus Aalykke, ${ }^{7}$ Thomas Ulstrup, ${ }^{7}$ Berit Schiøttz-Christensen, ${ }^{8}$ Hans Christian Horn, ${ }^{4}$ Amir Emamifar, ${ }^{1}$ Bent Duerlund, ${ }^{1}$ Lars Fischer, ${ }^{1}$ Inger Marie Jensen Hansen ${ }^{1,9}$

To cite: Andreasen RA, Kristensen LE, Ellingsen T, et al. Clinical characteristics of importance to outcome in patients with axial spondyloarthritis: protocol for a prospective descriptive and exploratory cohort study. BMJ Open 2017;7:e015536. doi:10.1136/ bmjopen-2016-015536

- Prepublication history for this paper is available online. To view these files please visit the journal online (http://dx.doi org/10.1136/bmjopen-2016015536).

Received 14 December 2016 Revised 9 May 2017 Accepted 6 June 2017

CrossMark

For numbered affiliations see end of article.

Correspondence to Inger Marie Jensen Hansen; inger.marie.jensen.hansen@ rsyd.dk

\section{ABSTRACT}

Introduction Spondyloarthritis $(\mathrm{SpA})$ is a heterogeneous spectrum of rheumatic diseases with either predominantly axial inflammatory symptoms of the spine and sacroiliac joints or predominantly peripheral arthritis. The two main entities of axial SpA (axSpA) are ankylosing spondylitis or non-radiographic axSpA (nr-axSpA). Tumour necrosis factor- $\alpha$ inhibitors have revolutionised the treatment of patients with axSpA who failed to respond to nonsteroidal anti-inflammatory drugs and physical therapy. Chronic pain is common in patients with SpA and may still persist despite the lack of signs of inflammation. This has led researchers to hypothesise that central pain sensitisation may play a role in the generation of chronic pain in SpA. The painDETECT Questionnaire (PDQ) is a screening tool developed to detect neuropathic pain components. The primary objective is to explore the prognostic value of the $\mathrm{PDQ}$ regarding treatment response in patients with axSpA 3 months after initiating a biological agent. Secondary aim is to evaluate the impact of extra-articular manifestations, comorbidities and patient-reported outcomes and elucidate if these factors influence treatment response.

Method and analysis We will include 60 participants ( $\geq 18$ years of age) diagnosed with axSpA independent of main entity, who initiate or switch treatment of a biologic. Data will be collected at baseline and at endpoint following Danish clinical practice ( $\geq 3$ months) of treatment with biologics. We will explore whether the PDQ and other phenotypical patient characteristics are prognostically important for response to biological therapy according to established response criteria like 50\% improvement in the Bath Ankylosing Spondylitis Disease Activity Index (50\%) and Ankylosing Spondylitis Disease Activity Score. Ethics and dissemination The study is approved by the Region of Southern Denmark's Ethics committee (S-20160094) and has been designed in cooperation with patient representatives. The study is registered at clinicaltrials.gov (NCT02948608, pre-results). Dissemination will occur through publication(s) in international peer-reviewed journal(s).
Strengths and limitations of this study

- To the best of our knowledge, this is the first prospective cohort study in an axial spondyloarthritis (axSpA) population, which examines whether various pain phenotypes are of prognostic importance.

- Focus on extra-articular manifestations and comorbidities.

- Involvement of several specialties and patient participation.

- The study is adding new knowledge to the research field within central sensitisation in patients with axSpA.

- Heterogeneity of the study population may limit our ability to infer from the analyses.

\section{INTRODUCTION}

Spondyloarthritis (SpA) are a heterogeneous group of chronic rheumatic diseases with overlapping symptoms including psoriatic arthritis (PsA), arthritis associated with inflammatory bowel disease (enteropathic arthritis), reactive arthritis, ankylosing spondylitis (AS) and undifferentiated SpA. ${ }^{1}$ Clinically, SpA can be dominated by peripheral joint involvement, classified as peripheral SpA, or by inflammatory back pain, classified as axial $\mathrm{SpA}(\operatorname{axSpA})$.

AxSpA is subdivided into two groups, referred to as non-radiographic and radiographic (ie, AS), respectively. The reported prevalence of axSpA worldwide ranges from $0.01 \%$ to approximately $2.5 \%$, and the incidence rates ranges between 0.48 and $63 / 100000 .^{2}$ Next to the spinal and articular symptoms, many patients with $\mathrm{SpA}$ also have extra-articular manifestations (EAM) which contribute to reduced quality of life. ${ }^{3}$ 
Acute anterior uveitis is a relatively common EAM in patients diagnosed with AS (9\%) and less frequent in patients diagnosed with another disease among the SpA spectrum. ${ }^{4}$ The aetiology of SpA is complex and not fully understood. It is known that $\mathrm{SpA}$ is associated with multiple genes, such as HLA-B2 $7^{5}$ although the pathogenesis of SpA remains largely unknown. The complexity of the disorder indicates a multifactorial aetiology involving multiple biological processes or pathways. ${ }^{6}$

There has been an increasing interest in how the gut microbiome may be the conductor or a mediator of the common inflammatory pathways seen in PsA and SpA..$^{7-9}$ The intestinal microbiome is able to affect extraintestinal distant sites, including the joints, through immunomodulation, as seen in arthritis associated with IBD. ${ }^{9}$ Certain types of bacterial gut infections, especially Yersinia enterocolitica, Shigella, Campylobacter jejuni and Salmonella typhimurium infections are associated with reactive arthritis. $^{1011}$

Calprotectin is a protein in neutrophil granulocytes and macrophages and was first found and described in $1980 .^{12}$ More studies focus on calprotectin measured in stool and plasma in IBD and confirm their value in diagnosis, disease activity evaluation, effect evaluation and relapse monitoring. ${ }^{13} 14$ A link between SpA and IBD has been established for decades. ${ }^{15}$ Even without clinical symptoms, up to $60 \%$ of the patients with AS present gut inflammation at colonoscopy, ${ }^{16}$ and a recent study found that nearly half of the patients with SpA had microscopic inflammatory lesions, with no differences between patients with peripheral and axSpA. ${ }^{17}$ A newly published study investigated the relation between serum and faecal levels of calprotectin and bowel histology in a SpA population and found that elevated serum calprotectin is significantly linked with bowel inflammation. ${ }^{18}$

Recently, nephrolithiasis (NL) was identified as a common (up to $10 \%$ ) and unrecognised extra-articular manifestation in AS. ${ }^{19}$ The risk of NL was more than twofold increased in patients with AS compared with the general population, partly explained by the altered intestinal absorption and bone-remodelling. Awareness of EAM among clinicians is important in view of their role in the diagnostic process, for treatment choices and for health-related quality of life.

Prognosis and as a consequence also management of pain in patients diagnosed with axSpA is a major clinical challenge. Pain may still persist despite the lack of signs of inflammation. This has led researchers to hypothesise that other than nociceptive pain may play a role in the generation of chronic pain in axSpA (ie, fibromyalgia/ central pain sensitisation). Fibromyalgia (using the 1990 ACR criteria for fibromyalgia) is a frequent comorbidity in patients with SpA, especially in the peripheral forms and with a female predominance. ${ }^{20}$ Fundamental knowledge of nociception from deep musculoskeletal structures and related mechanisms of sensitisation have been characterised in animals, but is still lacking in clinical sciences. ${ }^{21}$ Nociceptive and neuropathic components both contribute to pain. Since these components require different pain management strategies, correct pain diagnosis before and during treatment is highly desirable. As low back pain (LBP) patients constitute an important subgroup of chronic pain patients, a simple, patientbased questionnaires has been developed; painDETECT Questionnaire (PDQ). It can determine neuropathic pain components both in individual patients with LBP and in heterogeneous cohorts of such patients, with a high sensitivity, specificity and positive predictive accuracy. ${ }^{22}$ With the PDQ the rheumatologist may have a feasible and prognostically useful tool to predict the possible treatment outcome of anti-inflammatory treatment (ie, individualised therapy).

\section{Rationale and hypothesis}

The PDQ was developed and validated, for the purpose of establishing a screening tool to evaluate the likelihood of a neuropathic pain component being present in individual patients, in 2006. A validated algorithm was developed to be able to calculate a score with a range from 0 to 38. A score $\geq 19$ indicates that the presence of a neuropathic pain component is likely, whereas a score $\leq 12$ indicates that it is not. A score of 13-18 is considered uncertain. ${ }^{22}{ }^{23}$ To the best of our knowledge, only one small study has shown PDQ data in relation to axSpA. ${ }^{24}$ Their results suggest that back pain in AS is a complex pain condition that includes a neuropathic pain component.

We hypothesise that a PDQ score $\geq 19$ at baseline is of prognostic importance-potentially enabling prediction of a poorer patient reported treatment response according to the international standards- the Bath Ankylosing Spondylitis Disease Activity Index (BASDAI), the Bath Ankylosing Spondylitis Functional Index (BASFI), Ankylosing Spondylitis Disease Activity Score (ASDAS) when initiating or switching biological treatment in patients with axSpA.

\section{Objectives}

Our primary objective is to determine whether classification from the PDQ is valuable as a prognostic factor for treatment response in patients diagnosed with axSpA initiating or switching treatment with a biological agent. Secondary aim is to evaluate the impact of EAM, comorbidities evaluated by Charlson score, and patient-reported outcomes and elucidate if these factors-independently or by interaction-influence treatment responses. All participants will be screened for subclinical uveitis or signs of previously uveitis, which will provide us with an estimate of the prevalence of these conditions axSpA comorbidities in Denmark.

\section{Primary endpoint and key objective}

The key objective is to analyse the prognostic value of the PDQ score on the subsequent treatment response. The primary endpoint is the composite proportion of patients having achieved a BASDAI50 response or reduction of 
BASDAI $\geq 2 / 10$ after $\geq 3$ months' therapy with a biological agent.

\section{Key secondary objectives}

1. To analyse the proportion of patients having achieved an improvement of ASDAS $\geq 1$ after $\geq 3$ months' therapy with a biological agent.

2. To characterise and quantify the EAM according to Assessment of Spondyloarthritis International Society (ASAS) criteria.

3. To analyse the influence of self-reported disease activity at baseline as predictor of on treatment response: Estimation of disease activity and functional status is evaluated using BASDAI, BASFI, ASDAS and 36-Item Short-Form Health Survey (SF-36).

4. Change in swollen or tender joint count as determined by the 44-joint assessment at follow-up visit.

5. The Spondyloarthritis Research Consortium of Canada Enthesitis Index (SPARCC) at baseline and at follow-up visit.

6. The change in tender point count.

\section{Patient participation}

This study follows the European League Against Rheumatism (EULAR) recommendations for the inclusion of patient representatives in scientific projects. ${ }^{25}$ The study is designed with assistance from two Danish patient representatives, Bent Duerlund (BD) and Lars Kruse Fischer (LKF), and both are members of the author team as well. They are both diagnosed with AS and were selected in connection with a routine care visit. They have participated in discussion of relevance of the study, ethics and contributed with comments to the patient information. The primary investigator and the patient representatives will meet approximately every 6th month until the study ends.

\section{METHOD}

\section{Study design}

Patients with axSpA initiating or switching antirheumatic treatment (biologics) in routine care will be included as one group in this observational study. This study is designed as a 'closed cohort' with prospective enrolment of patients with axSpA over time. ${ }^{26}$ Information about the patients and their exposures will be collected at a single centre at two time points (figure 1), at baseline and after approximately 4 months of treatment according to the clinical standards in Denmark. Examinations will be carried out consecutively on the same day. Furthermore, there will be an eye examination carried out by an ophthalmologist, within 4 weeks from baseline visit. Participant inclusion is expected to run from November 2016 to June 2018. Patients fulfilling the eligibility criteria (see below) will be offered participation in this study.

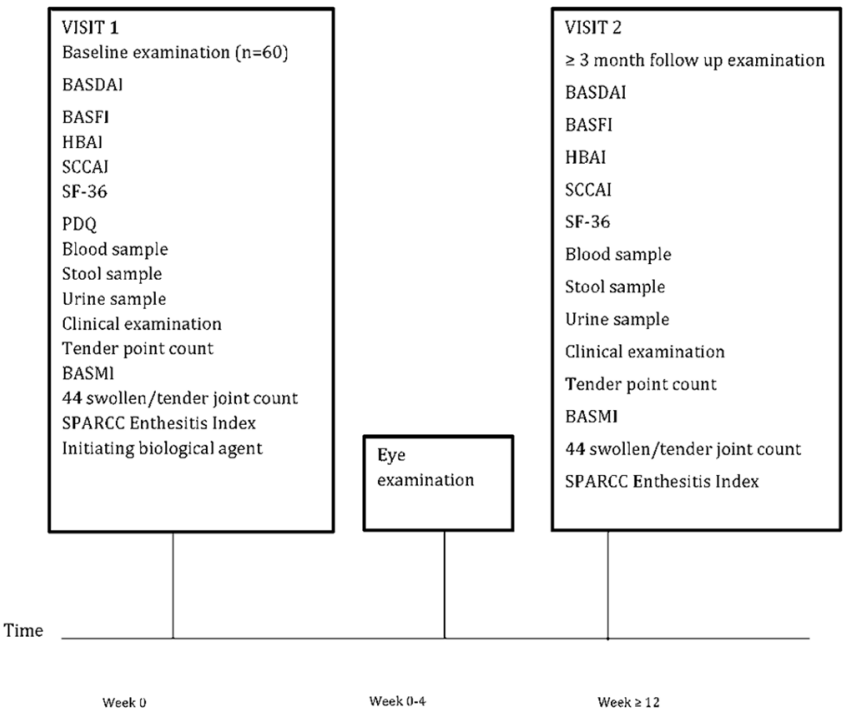

Figure 1 Participant timeline. BASDAI, Bath Ankylosing Spondylitis Disease Activity Index; BASFI, Bath Ankylosing Spondylitis Functional Index; HBAI, Harvey Bradshaw's Activity Index; PDQ, pain DETECT Questionnaire;

SCCAI, Simple Clinical Colitis Activity Index; SPARCC, Spondyloarthritis Research Consortium of Canada Enthesitis Score; VAS, Visual Analogue Scale; SF-36, 36-Item ShortForm Health Survey.

\section{Participants}

Participants will be recruited from The Department of Rheumatology, Odense University Hospital, Svendborg or Odense, Denmark and from The Spine Centre of Southern Denmark, Hospital Lillebaelt, Denmark. To be considered eligible for inclusion, participants must be $\geq 18$ years and diagnosed with axSpA and about to initiate or switch biological treatment. We use the ASAS criteria for axSpA (imaging arm) and the Modified New York criteria for AS, figure 2. Potential participants are identified by RAA, IMJH, HCH, TE, BS or site managers. The decision to initiate or change to biological treatment is taken collectively by senior rheumatologists at the department's biologics conference where representatives of the study are also present. Potential participants will be given oral and written information about the purpose and procedure of the study. Patients will be required to have normal results of a chest radiograph prior to the treatment with a biological agent and to have undergone screening for latent tuberculosis (TB) using the QuantiFERON TB Gold test. Patients in whom latent TB is discovered will require initiating therapy for $\mathrm{TB}$ prior to the first dose of the study agent. Patients are allowed to continue concurrent treatment with methotrexate (MTX), sulfasalazine, hydroxychloroquine, corticosteroids and non-steroidal anti-inflammatory drugs (NSAIDs) at stable doses during the study, though NSAID intake must be paused for a 14-day period within urine and stool sampling. Furthermore, potential participants with treatment failure on other biologics will be considered for inclusion (switching). Patients, who do not wish to participate, will be characterised by sex and age to assess any selection 


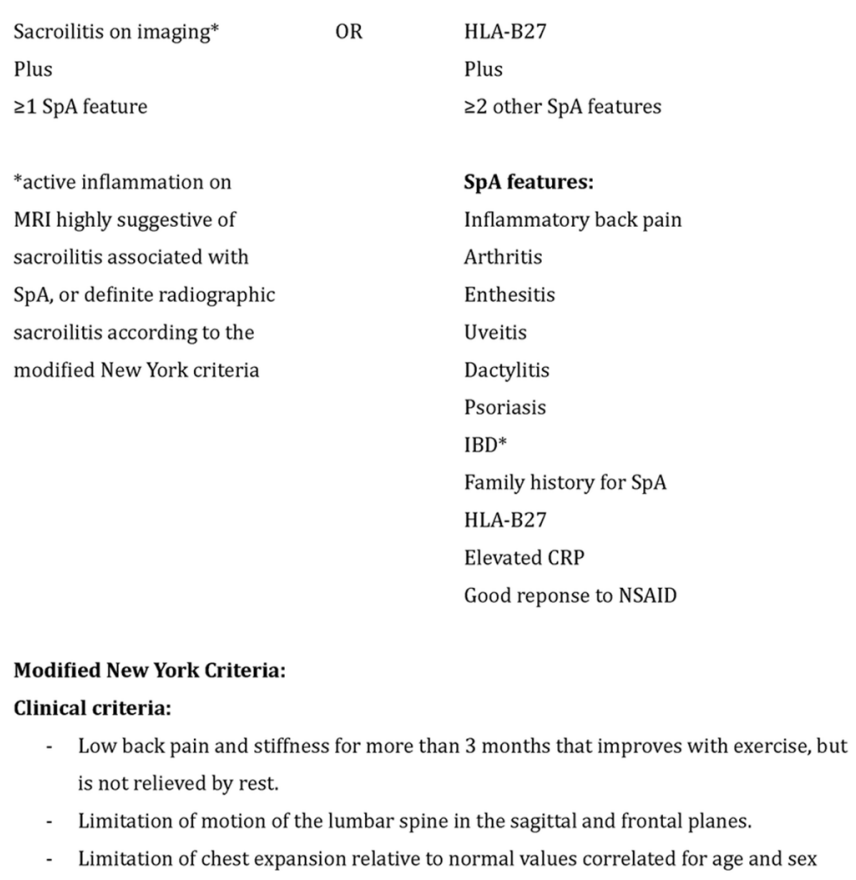

Radiological criterion:

- Sacroilitis grade $>2$ bilaterally or grade 3-4 unilaterally

Definite AS if the radiological criterion is associated with at least one clinical criterion.

Figure 2 ASAS classification criteria for axial SpA. AS, ankylosing spondylitis; ASAS, Assessment of Spondyloarthritis International Society; CRP, C reactive protein; NSAID, non-steroidal anti-inflammatory drug; SpA, spondyloarthritis.

bias. RAA and IMJH will screen potential participants regarding fulfilment of the inclusion criteria and none of the exclusion criteria. Furthermore, potential participants will be divided into two groups, A and B: group A: naive to any biologics (including patients newly diagnosed with axSpA), group B: switching therapy, figure 3 .

Inclusion criteria:

- Patients diagnosed with axSpA according to ASAS (imaging arm)

- $\geq 18$ of age at time of consent

- Ability and willingness to give written informed consent and to meet the requirements of this protocol

- Patients must have a history of active disease and a BASDAI >40 (10-100) despite current or previous NSAIDs therapy

Exclusion criteria

- Age $<18$ years

- BASDAI $\leq 40$

- No consent

- Pregnancy

- Active or latent TB

- Diagnosed HIV

- Diagnosed hepatitis

- Current or past malignant disease

- Recurrent or chronic infection (viral, fungal or bacterial)

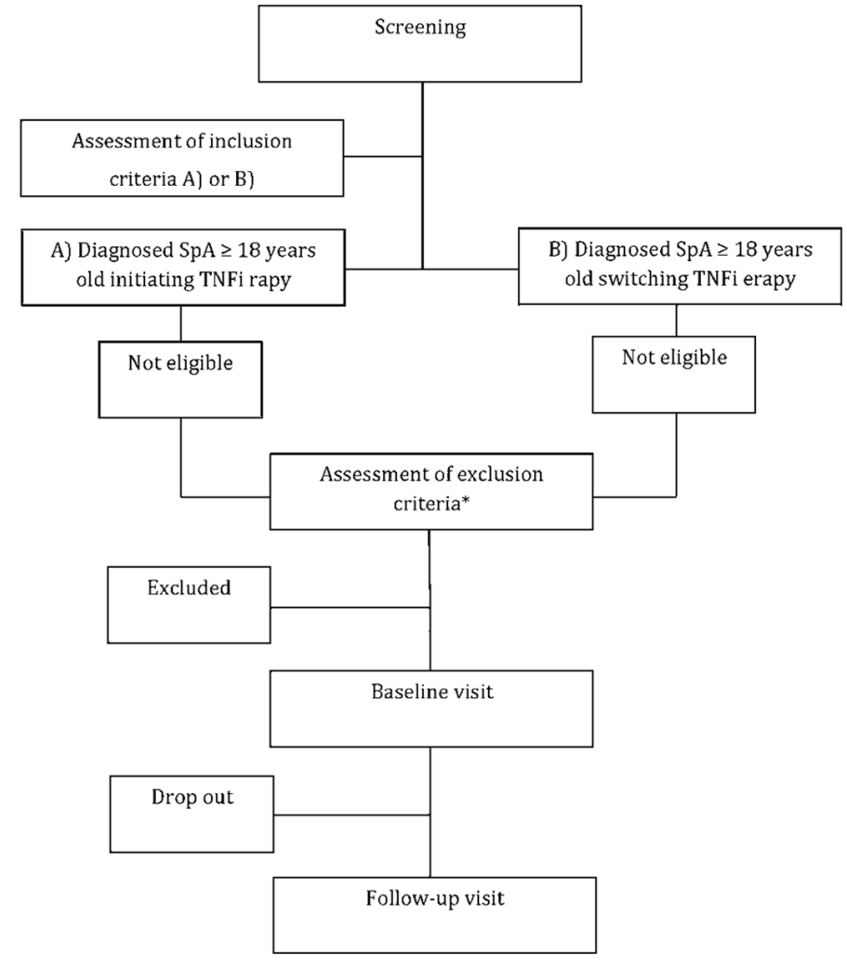

*Exclusion criteria: (1) Age < 18 years; (2) No consent; (3) Pregnancy; (4) Active or latent TB; (5) Human immunodeficiency virus; (6) Hepatitis; (7) Current or past malignant disease; (8) Recurrent or chronic infection (viral, fungal or bacterial); (9) Multiple sclerosis; (10) Heart failure (NYHA class III/IV)

Figure 3 Overview of participant flow. SpA, spondyloarthritis; TB, tuberculosis; TNFi, tumour necrosis factor- $\alpha$ inhibitors.

- Multiple sclerosis

- Heart failure (New York Heart Association (NYHA) class III/IV)

\section{Treatment}

Following the baseline examination, all enrolled participants will be treated in accordance with the clinical treatment guideline developed by The Danish Council for the use of expensive hospital medicines (RADS). Currently (while writing the protocol), first-line therapy is a biosimilar infliximab compound (Remsima, Orion Pharma); infliximab is a human-murine chimeric monoclonal antibody directed against tumour necrosis factor $(\mathrm{TNF})^{27}$ and is administrated at a dose of $5 \mathrm{mg} / \mathrm{kg}$ via intravenous infusion at weeks $0,2,6$ and then every 8 weeks. If the treatment is not tolerated, it will be stopped.

As part of clinical practice, patients are allowed to continue their conventional disease-modifying antirheumatic drugs: MTX and/or sulfasalazine. In accordance with Danish guidelines for intervention strategy patients will be treated with intra-articular glucocorticoid injections if needed during follow-up. Blood sample during treatment will be performed in accordance with the current clinical practice. 


\section{Eye examination}

The eye examination will be performed by an ophthalmologist in accordance with normal clinical practice in Denmark. The following symptoms of acute anterior uveitis are registered: pain, redness of the globe, photophobia and reduced visual acuity. Best-corrected visual acuity as determined by Snellen, intraocular pressure, slit-lamp examination and dilated fundus examination are performed and previous signs of anterior uveitis, for example, keratic precipitates and posterior synechiae are recorded. Ocular inflammation is graded according to the Standardisation of Uveitis Nomenclature working group recommendations. ${ }^{28}$

\section{Variables and outcome measures}

Included patients will undergo an examination programme to collect variables shown in table 1 .

Information on ${ }^{1}$ demographic data (age, sex, height, weight, educational level, smoking status), ${ }^{2}{ }^{29}$ clinical characteristics (symptom duration, duration since axSpA diagnosis and type of the form of clinical involvement), HLA-B27, family history and $\mathrm{EAM}^{3}$ activity data and monitoring methods (erythrocyte sedimentation rate, $\mathrm{C}$ reactive protein (CRP), Visual Analogue Scale (VAS) for pain and activity, morning stiffness, joint assessment, painful enthesis (BASDAI)), ${ }^{4}$ employment status and functional ability (BASFI), PDQ tender point count and SF- $36^{5}$ comorbidity (including depression) will be obtained by interview and clinical examination at baseline and follow-up visit (Charlson Comorbidity Index Score),${ }^{630}$ we will use the Simple Clinical Colitis Activity Index (SCCAI) and the Harvey Bradshaw's Activity Index (HBAI) regarding symptoms of inflammatory bowel diease (IBD).

\section{Clinical examination}

A trained healthcare professional will perform the interviews and clinical examinations. During the study period, the patients will receive routine care, with prospective registration in DANBIO (a nationwide registry, approved as a clinical quality registry by the Danish National Board of Health), according to normal clinical practice in Denmark.

The SPARCC ${ }^{31}$ will be used to enthesitis count.

\section{Assessment of pain}

The PDQ has been translated into 19 different languages, including Danish. It is composed of questions regarding pain intensity (three numeric rating scales, pain course pattern, a pain drawing reflecting pain radiation and seven questions addressing somatosensory phenomena which the patient rates on a six-category Likert scale (never-very strongly). A score ranging between 0 and 38 , based on the patient's answers in the questionnaire, is calculated. For diagnostic purposes, a validated algorithm has been developed. A painDETECT score $\geq 19$ indicates that a neuropathic pain component is likely, a score of $13-18$ is considered uncertain and a score $\leq 12$ indicates that a neuropathic pain component is unlikely, resulting in three categories of patient pain characteristics. For a comprehensive description and overview of the single questions (items) in the questionnaire, we refer to the original article by Freynhagen $e t a .^{22}$ The tender point count, a part of the 1990 ACR criteria for fibromyalgia, where the cut-off is 11 of 18 tender points, ${ }^{32}$ will be used as well.

\section{Blood, urine and stool samples}

Faecal and plasma calprotectin ${ }^{33}$ and orosomucoid ${ }^{34}$ will be assessed at baseline and follow-up visit and will be considered as an indicator for intestinal inflammation. Furthermore, we will examine the stool for intestinal pathogen bacteria. A urine sample will be assessed at baseline and follow-up visit. Any event of NL will be registered. Any intake of NSAIDs will be paused in a 14-day period prior to baseline visit.

Blood samples (table 1) will be collected and analysed according to standard procedures. Blood sample during treatment will be performed in accordance with the current clinical practice. The frequency of these blood samples will be decided by the responsible treating rheumatologist depending on symptoms or signs of side effects. The exact number of tests and the precise time line for performing test may therefore deviate from the one scheduled in figure 3. Furthermore, a Biobank will be established in connection with the routine blood samples taken at baseline $(\sim 90 \mathrm{~mL})$. The blood samples will be handled and registered by research laboratory technicians at the laboratory department and stored at Odense University Hospital (in the cold store). The material was collected in order to carry out analyses in connection with the current investigation and for future research in SpA.

\section{Patient-reported outcomes}

In the context of monitoring disease activity in patients with $\mathrm{SpA}$, most of the variables are based on patient-reported outcomes. We will use the following in our study:

1. The BASDAI: The BASDAI is a 6-item scale to measure severity of fatigue, spinal and peripheral joint pain, localised tenderness and morning stiffness, using the VAS.

2. The BASFI: The BASFI is a 10-item scale on which respondents rate the degree of difficulty they have in performing certain tasks, using VAS from 0 (easy) to 100 (impossible). The mean of the 10 responses is the BASFI score.

3. The Medical Outcomes Study SF-36 is a generic health status questionnaire that was developed as a tool to compare various aspects of health status across a general and broad patient population. ${ }^{35-37}$ The SF-36 examines eight general health domains: physical functioning, role limitations due to physical health problems, bodily pain, general health perceptions, vitality, social functioning, role limitations due to emotional problems and 
Table 1 Summary of measures to be collected

\section{Demographics}

\begin{tabular}{|c|c|c|}
\hline $\operatorname{Sex}(M / F)$ & $x$ & \\
\hline Height (m) & $x$ & \\
\hline BMI $\left(\mathrm{kg} / \mathrm{m}^{2}\right)$ & $\mathrm{x}$ & $x$ \\
\hline Education level & $\mathrm{x}$ & \\
\hline \multicolumn{3}{|l|}{ Disease characteristics } \\
\hline Symptom duration (months) & $X$ & \\
\hline Symptom duration prior to diagnosis (months) & $\mathrm{x}$ & \\
\hline Axial disease (yes/no) & $\mathrm{x}$ & \\
\hline Current NSAID at assessment (yes/no) & $\mathrm{x}$ & $x$ \\
\hline Prior DMARD therapy (yes/no) & $X$ & \\
\hline MTX dose (mg/week) & $\mathrm{X}$ & $x$ \\
\hline Other current DMARD therapy & $X$ & $X$ \\
\hline No of previous biologics used (if any) & $X$ & \\
\hline Name of current biological agent & $X$ & $X$ \\
\hline Dose of prednisolone orally at assessment (mg/week) & $X$ & $X$ \\
\hline Dose of prednisolone orally 1 week prior to assessment (mg/week) & $X$ & $X$ \\
\hline Dose of prednisolone orally 2 weeks prior to assessment (mg/week) & $X$ & $x$ \\
\hline Tender points & $x$ & $x$ \\
\hline Eye examination & $x$ & $x$ \\
\hline
\end{tabular}

SpA features

\begin{tabular}{|c|c|c|}
\hline Anterior uveitis, past or present (yes/no) & $\mathrm{X}$ & $\mathrm{X}$ \\
\hline Inflammatory bowel disease (IBD), past or present (yes/no) & $\mathrm{X}$ & $\mathrm{X}$ \\
\hline Psoriasis, past or present (yes/no) & $\mathrm{X}$ & $\mathrm{X}$ \\
\hline Psoriatic nail lesions (yes/no) & $\mathrm{X}$ & $\mathrm{X}$ \\
\hline Preceding infection (yes/no) & $\mathrm{X}$ & $\mathrm{X}$ \\
\hline Total enthesitis count & $\mathrm{X}$ & $\mathrm{X}$ \\
\hline Dactylitis count & $\mathrm{x}$ & $\mathrm{x}$ \\
\hline Comorbidities & $\mathrm{X}$ & $\mathrm{X}$ \\
\hline Depression past or present & $\mathrm{x}$ & $\mathrm{x}$ \\
\hline \multicolumn{3}{|l|}{ Charlson comorbidity index score } \\
\hline Basic metabolic screening & $x$ & $x$ \\
\hline S-creatinin, S-calcium, S-urat, S-sodium, S-potassium & $\mathrm{X}$ & $X$ \\
\hline Jrine samples & $X$ & $X$ \\
\hline
\end{tabular}




\section{Table 1 Continued}

\section{Demographics}

Urine-pH (dip-stick), U-calcium, U-chloride, U-potassium, $X$

$\mathrm{X}$

U-sodium, U-albumin, U-oxalate

\section{Patient-reported outcomes}

BASDAI, BASFI $\quad X \quad X$

\section{SF-36}

$\begin{array}{lll}\text { PDQ score } & X & X \\ \text { HAQ score } & X & X \\ \text { VAS fatigue, VAS pain, VAS global } & X & X \\ \text { SCCAI } & X & X \\ \text { HBAI } & X & X \\ \text { Blood samples } & X & X \\ \text { HLA-B27 (positive/negative) } & X & X\end{array}$

CRP, ESR, orosomucoid, vitamin D, immunoglobulin A, ALAT, alkaline phosphatise, creatinine, estimated GFR, haemoglobin, erythrocyte volume fraction, MCHC, MCV, leucocytes, differential count, thrombocyte, LDL- cholesterol, HDL-cholesterol, total cholesterol, serology test for shigella, plasma calprotectin, IgA†

Stool sample $\mathrm{X}$

Faecal calprotectin (mg/L) $\mathrm{x}$ $X$

Yersinia enterocolitica, Shigella, Campylobacter jejuni, Salmonella typhimurium

$\begin{array}{ccc}\text { Chlamydia testing } & X & X \\ \text { Composite outcome measures } & X & X \\ \text { BASDAI50 response, } \triangle \text { ASDAS } & X & X \\ \end{array}$

\section{Activity Score}

*Will not be repeated if already taken.

†Will not be repeated if taken within the past 2 weeks.

BMI, body mass index; ALAT, alanine transaminase; ASDAS, ankylosing spondylitis disease; BASDAI, Bath Ankylosing Spondylitis Disease Activity Index; BASFI, Bath Ankylosing Spondylitis Functional Index; HAQ, health assessment questionnaire CRP, C reactive protein; DMARD, disease-modifying antirheumatic drugs; ESR, erythrocyte sedimentation rate; GFR, glomerular filtration rate; HBAl, Harvey Bradshaw's Activity Index; IgA, immunoglobulin A; MCHC, mean corpuscular haemoglobin concentration; MCV, mean corpuscular volume; LDL, lowdensity lipoprotein; HDL, high-density lipoprotein, MTX, methotrexate; NSAID, non-steroidal anti-inflammatory drug; PDQ, painDETECT Questionnaire; SCCAI, Simple Clinical Colitis Activity Index; BASMI, Bath Ankylosing Spondylitis Metrology Index, SPARCC, Spondyloarthritis Research Consortium of Canada Enthesitis Score; VAS, Visual Analogue Scale; SF-36, 36-Item Short-Form Health Survey; SpA, spondyloarthritis.

mental health. Furthermore, a physical and mental component summary score can be calculated. We will use the Danish version of SF- $36^{38}$ which uses a 4-week recall period.

4. Disease activity in patients with ulcerative colitis is assessed by the SCCAI - a score consisting of five questions regarding bowel frequency day/night, urgency of defecation, blood in stool and general well-being. ${ }^{39}$

5. Disease activity in patients with Crohn's disease is assessed by the HBAI-consisting of five clinical parameters including number of liquid stools per day, abdominal pain, abdominal mass, general wellbeing and extraintestinal manifestations including arthralgia. $^{40}$

\section{Exploratory outcome measures and response criteria}

The response to the treatment at follow-up visit will be assessed by various patient-reported outcome measures. The BASDAI50 response criteria and ASDAS response criteria will be used to define treatment response. Patients who will not respond to TNF- $\alpha$ blocking therapy after 4 months will be classified as primary non-responders. The ASDAS is a composite index that assesses the disease activity. The ASDAS incorporates three items from the BASDAI: back pain (VAS), duration of morning stiffness (VAS) and pain of peripheral joints (VAS), as well as patient global assessment of the disease activity (VAS) and CRP $(\mathrm{mg} / \mathrm{L}) .{ }^{41} 42 \mathrm{An}$ ASDAS $<1.3$ is defined as inactive disease, ASDAS $\geq 1.3$ to $<2.1$ is moderate disease activity, ASDAS $\geq 2.1$ to $\leq 3.5$ is high disease activity and ASDAS $\geq$ is very high disease activity. ${ }^{434}$ 


\section{STATISTICAL METHODS}

\section{Sample size and power considerations}

For a comparison of two independent binomial proportions (high vs other PDQ category) using Pearson's $\chi^{2}$ statistic with a $\chi^{2}$ approximation with a two-sided significance level of 0.05 , a total sample size of 54 patients with SpA assuming that the proportion of patients with a high PDQ of $50 \%$ achieves a statistical power of at least $80 \%$ when the proportions having a BASDAI response are $15 \%$ and $50 \%$, in high versus other PDQ category.

Thus, we aim to include 60 patients in total (anticipating 30 patients will have a $\mathrm{PDQ}>19$ ), corresponding to an approximate power of $84.3 \%$ when the proportions with a BASDAI-response are $15 \%$ and $50 \%$, respectively.

\section{Statistical analysis}

All descriptive statistics and tests will be reported in accordance with the recommendations of the 'Enhancing the QUAlity and Transparency Of health Research' network: $:^{45}$ the STROBE Statement. ${ }^{26}$ Baseline variables will be described for all participants, for all continuous variables with means (and SDs) or medians (and IQRs) and for categorical variables percentages and frequencies will be reported. For comparisons, $\chi^{2}$ will be used for categorical variables and

the Mann-Whitney test for continuous data. To assess the bivariate association between variables, correlation will be applied using Spearman's rank correlation coefficient.

For the statistical testing, we will consider $p$ values less than 0.05 (and 95\% CIs excluding the null) to be statistically significant. Missing data at follow-up will be imputed by use of a non-responder assumption (applying baseline observation carried forward for continuous data).

To study the prognostic value of the PDQ score in relation to treatment response, regression models will be applied to study if pain measurement and/or other demographic/clinical measures have an impact on the treatment response measures and subsequently multivariable regression models will be applied if deemed necessary. According to the principles stated in the STROBE statement both crude and adjusted estimates will be reported.

\section{Discussion}

The proposed study attempts to evaluate the pain mechanism, characteristics of the patients regarding EAM, treatment response as well as clinical activity in patients with axSpA. The study will contribute to the understanding of the important role of central pain components in patients with axSpA by determining the prognostic value of $P D Q$ score regarding treatment response. We will describe the relationship between central sensitisation and subsequent treatment outcome. Knowledge about the presence of a neuropathic pain component may be particularly important to the rheumatologists treating patients with axSpA, since the road to biological treatment is short and a high BASDAI score primarily derived from patient-reported information. Identifying which patients with axSpA who are likely to benefit from biologics (eg, TNF- $\alpha$ inhibitors) therapy is important, especially in view of the costs and potential side effects of these agents.

We anticipate (and hope) that this study can contribute to set focus on the need of treating the underlying pain mechanisms which not can be explained by inflammatory genesis in patients with axSpA. The patients will probably have a better prognosis, if they are educated in and informed of all the various factors (ie, EAM and comorbidities) that can influence on the overall quality of life and treatment response. IBD is a frequent EAM among patients with SpA and is important to take into account regarding treatment strategy, as NSAIDs increases the risk of flare in IBD. Using calprotectin measurements in stool and serum in this study may provide a promising strategy to identify patients with axSpA at risk of bowel inflammation and could play a role in overall patient stratification.

We know there is a higher prevalence of comorbidities in patients with inflammatory joint diseases compared with the general population, ${ }^{46}{ }^{47}$ but the patients may receive suboptimal medical treatment for the comorbidities compared with the general population, possibly due to the focus on their rheumatic diseases or because the lack of consensus of which health professional should take the responsibility for the patient as a whole. In this study, we outline the patient's comorbidities which are in accordance with EULAR proposal regarding points to consider to collect comorbidities in patients with chronic inflammatory rheumatic diseases. ${ }^{48}$

The study may be limited by sample size possible, based on the anticipation that these patients (with axSpA) represent a heterogeneous population which may limit our ability to infer from the analyses.

In conclusion, the results of this study may add knowledge to the current black box of complexities around disease measures and prognosis and the efficacy of biologics among patients diagnosed with axSpA. Hopefully, this project will facilitate a more efficient use of expensive drugs as well as multidisciplinary rehabilitation, by revealing the need for a more individualised biopsychosocial model in the future management strategy.

\section{ETHICS AND DISSEMINATION}

The study has been approved by the Region of Southern Denmark's Ethics Committee with identification number S-20160094. Signed informed consent will be obtained from all participants. The study is registered at Clinicaltrials.gov (NCT02948608). We aim to disseminate the results of the proposed study through international conferences and international peer-reviewed journal(s).

Author affiliations

${ }^{1}$ Department of Medicine, Section of Rheumatology, Odense University Hospital, Svendborg, Denmark 
${ }^{2}$ Musculoskeletal Statistics Unit, The Parker Institute, Bispebjerg and Frederiksberg Hospital, Copenhagen, Denmark

${ }^{3}$ Patient Data Explorative Network (OPEN), Odense University Hospital, Odense, Denmark

${ }^{4}$ Department of Rheumatology, Odense University Hospital, Odense, Denmark ${ }^{5}$ Rheumazentrum Ruhrgebiet Herne, Ruhr-University Bochum, Bochum, Germany

${ }^{6}$ Department of Ophthalmology, Odense University Hospital, Odense, Denmark ${ }^{7}$ Department of Medicine, Section of Gastroenterology, Odense University Hospital, Svendborg, Denmark

${ }^{8}$ Department of Spine Centre of Southern Denmark, Hospital Lillebaelt, Institute of Regional Health Research, University of Southern Denmark, Middelfart, Denmark ${ }^{9}$ Department of Clinical Research, University of Southern Denmark, Odense, Denmark

Acknowledgements The authors wish to acknowledge the help from all participating patients and contributors of the staff members of the Department of Rheumatology, Odense University Hospital, Svendborg/Odense, the Department of Ophthalmology, Odense University Hospital, Spine Centre of Southern Denmark, Hospital Lillebaelt and The Department of Clinical Biochemistry, Odense University Hospital, Svendborg.

Contributors RAA serves as the principal investigator for this study. RC, LEK, TE, IMJH, BD and LF participated in the design of the study and drafting of the protocol. $\mathrm{BS} \emptyset-\mathrm{C}, \mathrm{HCH}, \mathrm{CA}, \mathrm{TU}, \mathrm{AE}, \mathrm{JW}$ and $\mathrm{XB}$ have all revised the protocol for intellectual content. XB has made expert opinion on outcome measures in the study. CA and TU have provided expert opinions on various aspects on inflammatory bowel disease. $\mathrm{RC}$ has provided expert opinion concerning research design and subsequent statistics. JW has provided expert opinion concerning the eye examination and is responsible for the eye examination. $\mathrm{BS} \emptyset-\mathrm{C}, \mathrm{HCH}, \mathrm{CA}, \mathrm{AE}$ and $\mathrm{TU}$ will assist with recruitment of patients. IMJH has been the main supervisor for all aspects of the protocol. RAA is taking the responsibility for the integrity of the protocol manuscript and approves the final version for publication.

Funding The study is supported by grants from (1) The Faculty of Health Sciences, University of Southern Denmark, (2) Carl Hansen \& Son, (3) The Department of Medicine, Odense University Hospital, Svendborg, (4) The Region of Southern Denmark, (5) The Danish Rheumatism Association, (6) Erna Hamiltons fond, (7) The Parker Institute, Bisbjerg and Frederiksberg Hospital is supported by a core grant from the Oak Foundation.

Competing interests RAA, LEK, TE, TU, AE, BD, LF and IMJH have nothing to disclose. $R C$ reports non-financial support from Board membership, grants from Consultancy (AbbVie, Amgen, Axellus A/S, Bristol-Myers Squibb, Cambridge Weight Plan, Celgene, Eli Lilly, Hospira, MSD, Norpharma, Novartis, Orkla Health, Pfizer, Roche, Sobi, Takeda), personal fees from Employment (Research Unit for Musculoskeletal Function and Physiotherapy, Institute of Sports Science and Clinical Biomechanics, University of Southern Denmark), non-financial support from Expert testimony, grants from Grants/grants pending (Axellus A/S, AbbVie, Cambridge Weight Plan, Janssen, MSD, Mundipharma, Novartis, and Roche), grants from Payment for lectures including service on speakers bureaus (Abbott, Amgen, Axellus, Bayer HealthCare Pharmaceuticals, Biogen Idec, Bristol-Myers Squibb, Cambridge Weight Plan, Ipsen, Janssen, Laboratoires Expanscience, MSD, Mundipharma, Norpharma, Novartis, Pfizer, Roche, Rottapharm-Madaus, Sobi, and Wyeth), grants from Payment for manuscript preparation (Axellus, Bristol-Myers Squibb, and Cambridge Weight Plan, Aleris-Hamlet (via Norpharma)), non-financial support from Patents (planned, pending or issued), non financial support from Royalties, grants from Payment for development of educational presentations (Bristol-Myers Squibb, MSD, Pfizer), non-financial support from Stock/stock options, grants from Travel/accommodations/meeting expenses unrelated to activities listed (Abbott, AbbVie, Axellus, Bristol-Myers Squibb, Cambridge Weight Plan, Celgene, Laboratoires Expanscience, Norpharma, Novartis, Pfizer, Roche, RottapharmMadaus, and Wyeth), non-financial support from Other (err on the side of full disclosure), outside the submitted work; and he is involved in many healthcare initiatives and research that could benefit from wide uptake of this publication (including Cochrane, OMERACT, IDEOM, RADS and the GRADE Working Group). The Parker Institute is supported by a core grant from the Oak Foundation; The Oak Foundation is a group of philanthropic organizations that, since its establishment in 1983 , has given grants to not-for-profit organizations around the world. JW reports personal fees from Abbvie (advisory board), personal fees from Santhera (speaker), grants from Bayer (AAO Congres invitation), outside the submitted Work. CA reports personal fees from Boardmembership (Ferring, Takeda), personal fees from Payment for lectures i (Abbott, Ferring, Norgine, MSD, Takeda), grants from Travel/ accommodations/meeting expenses unrelated to activities listed (Abvie, Ferring, Eli
Lilly, Norgine, Medtronics, Takeda,Tillots) , outside the submitted work. BC reports personal fees from Pfizer, outside the submitted work. $\mathrm{HCH}$ reports personal fees from Advisory Board Abbvie Denmark, psoriatic arthritis, spondyloarthritis and uveitis, personal fees from Advisory Board Novartis Denmark, non-financial (support from Travel/accomodation/meeting expenses unrelated to activity listed Abbvie, Pfizer, BMS, MSD), grants from Payment for educational presentations (Novartis Denmark), outside the submitted work.

Provenance and peer review Not commissioned; externally peer reviewed.

Open Access This is an Open Access article distributed in accordance with the Creative Commons Attribution Non Commercial (CC BY-NC 4.0) license, which permits others to distribute, remix, adapt, build upon this work non-commercially, and license their derivative works on different terms, provided the original work is properly cited and the use is non-commercial. See: http://creativecommons.org/ licenses/by-nc/4.0/

(c) Article author(s) (or their employer(s) unless otherwise stated in the text of the article) 2017. All rights reserved. No commercial use is permitted unless otherwise expressly granted.

\section{REFERENCES}

1. van der Horst-Bruinsma IE, Nurmohamed MT. Management and evaluation of extra-articular manifestations in spondyloarthritis. Ther Adv Musculoskelet Dis 2012;4:413-22.

2. Stolwijk $C$, Boonen $A$, van Tubergen $A$, et al. Epidemiology of spondyloarthritis. Rheum Dis Clin North Am 2012;38:441-76.

3. Braun J, Kiltz U, Baraliakos X, et al. Optimisation of rheumatology assessments - the actual situation in axial spondyloarthritis including ankylosing spondylitis. Clin Exp Rheumatol 2014;32(5 Suppl 85):S96-104.

4. Mitulescu TC, Popescu C, Naie A, et al. Acute anterior uveitis and other extra-articular manifestations of spondyloarthritis. J Med Life 2015;8:319-25.

5. Zhao J, Chen J, Yang TH, et al. Insights into the pathogenesis of axial spondyloarthropathy from network and pathway analysis. BMC Syst Biol 2012;6 (Suppl 1):S4.

6. Olivieri I, van Tubergen A, Salvarani C, et al. Seronegative spondyloarthritides. Best Pract Res Clin Rheumatol 2002;16:723-39.

7. Eppinga $\mathrm{H}$, Konstantinov SR, Peppelenbosch MP, et al. The microbiome and psoriatic arthritis. Curr Rheumatol Rep 2014;16:407.

8. Kabeerdoss J, Sandhya P, Danda D. Gut inflammation and microbiome in spondyloarthritis. Rheumatol Int 2016;36:457-68.

9. Asquith M, Elewaut D, Lin P, et al. The role of the gut and microbes in the pathogenesis of spondyloarthritis. Best Pract Res Clin Rheumatol 2014;28:687-702.

10. Jacques $P$, Elewaut $D$. Joint expedition: linking gut inflammation to arthritis. Mucosal Immunol 2008;1:364-71.

11. Nielsen LN, Sheppard SK, McCarthy ND, et al. MLST clustering of Campylobacter jejuni isolates from patients with gastroenteritis, reactive arthritis and Guillain-Barré syndrome. J Appl Microbiol 2010;108:591-9.

12. Røseth AG, Fagerhol MK, Aadland E, et al. Assessment of the neutrophil dominating protein calprotectin in feces. A methodologic study. Scand J Gastroenterol 1992;27:793-8

13. Smith LA, Gaya DR. Utility of faecal calprotectin analysis in adult inflammatory bowel disease. World J Gastroenterol 2012;18:6782-9.

14. Kalla R, Kennedy NA, Ventham NT, et al. Serum calprotectin: a novel diagnostic and prognostic marker in inflammatory bowel diseases. Am J Gastroenterol 2016;111:1796-805.

15. Stolwijk C, Essers I, van Tubergen A, et al. The epidemiology of extra-articular manifestations in ankylosing spondylitis: a populationbased matched cohort study. Ann Rheum Dis 2015;74:1373-8.

16. Van Praet $L$, Jans $L$, Carron $P$, et al. Degree of bone marrow oedema in sacroiliac joints of patients with axial spondyloarthritis is linked to gut inflammation and male sex: results from the GIANT cohort. Ann Rheum Dis 2014;73:1186-9.

17. Van Praet L, Van den Bosch FE, Jacques $P$, et al. Microscopic gut inflammation in axial spondyloarthritis: a multiparametric predictive model. Ann Rheum Dis 2013;72:414-7.

18. Cypers H, Varkas G, Beeckman S, et al. Elevated calprotectin levels reveal bowel inflammation in spondyloarthritis. Ann Rheum Dis 2016;75:1357-62.

19. Jakobsen AK, Jacobsson LT, Patschan O, et al. Is nephrolithiasis an unrecognized extra-articular manifestation in ankylosing spondylitis? A prospective population-based Swedish national cohort study with matched general population comparator subjects. PLoS One 2014;9:e113602. 
20. Wach J, Letroublon MC, Coury F, et al. Fibromyalgia in Spondyloarthritis: Effect on Disease Activity Assessment in Clinical Practice. J Rheumatol 2016;43:2056-63.

21. Arendt-Nielsen L, Graven-Nielsen T. Translational musculoskeletal pain research. Best Pract Res Clin Rheumatol 2011;25:209-26.

22. Freynhagen R, Baron R, Gockel U, et al. painDETECT: a new screening questionnaire to identify neuropathic components in patients with back pain. Curr Med Res Opin 2006;22:1911-20.

23. Christensen AW, Rifbjerg-Madsen S, Christensen R, et al. Nonnociceptive pain in rheumatoid arthritis is frequent and affects disease activity estimation: cross-sectional data from the FRAME study. Scand J Rheumatol 2016;45:461-9.

24. Wu Q, Inman RD, Davis KD. Neuropathic pain in ankylosing spondylitis: a psychophysics and brain imaging study. Arthritis Rheum 2013;65:1494-503.

25. de Wit MP, Berlo SE, Aanerud GJ, et al. European League Against Rheumatism recommendations for the inclusion of patient representatives in scientific projects. Ann Rheum Dis 2011;70:722-6.

26. Vandenbroucke JP, von Elm E, Altman DG, et al. Strengthening the Reporting of Observational Studies in Epidemiology (STROBE): explanation and elaboration. Int J Surg 2014;12:1500-24.

27. Park W, Yoo DH, Jaworski J, et al. Comparable long-term efficacy, as assessed by patient-reported outcomes, safety and pharmacokinetics, of CT-P13 and reference infliximab in patients with ankylosing spondylitis: 54-week results from the randomized, parallel-group PLANETAS study. Arthritis Res Ther 2016;18:25.

28. Jabs DA, Nussenblatt RB, Rosenbaum JT. Standardization of Uveitis Nomenclature (SUN) Working Group. Standardization of uveitis nomenclature for reporting clinical data. Results of the First International Workshop. Am J Ophthalmol 2005;140:509-16.

29. Højgaard P, Glintborg B, Hetland ML, et al. Association between tobacco smoking and response to tumour necrosis factor $\alpha$ inhibitor treatment in psoriatic arthritis: results from the DANBIO registry. Ann Rheum Dis 2015;74:2130-6.

30. Charlson ME, Pompei P, Ales KL, et al. A new method of classifying prognostic comorbidity in longitudinal studies: development and validation. J Chronic Dis 1987;40:373-83.

31. Maksymowych WP, Mallon C, Morrow S, et al. Development and validation of the Spondyloarthritis Research Consortium of Canada (SPARCC) Enthesitis Index. Ann Rheum Dis 2009;68:948-53.

32. Wolfe F, Smythe HA, Yunus MB, et al. The American College of Rheumatology 1990 Criteria for the Classification of Fibromyalgia. Report of the multicenter criteria committee. Arthritis Rheum 1990;33:160-72.

33. Sipponen T, Kolho KL. Fecal calprotectin in diagnosis and clinical assessment of inflammatory bowel disease. Scand J Gastroenterol 2015;50:74-80.

34. Hochepied T, Berger FG, Baumann H, et al. Alpha(1)-acid glycoprotein: an acute phase protein with inflammatory and immunomodulating properties. Cytokine Growth Factor Rev 2003;14:25-34.

35. McHorney CA, Ware JE, Lu JF, et al. The MOS 36-item ShortForm Health Survey (SF-36): III. Tests of data quality, scaling assumptions, and reliability across diverse patient groups. Med Care 1994;32:40-66

36. McHorney CA, Ware JE, Raczek AE. The MOS 36-Item Short-Form Health Survey (SF-36): II. Psychometric and clinical tests of validity in measuring physical and mental health constructs. Med Care 1993;31:247-63.

37. Ware JE, Sherbourne CD. The MOS 36-item short-form health survey (SF-36). I. Conceptual framework and item selection. Med Care 1992;30:473-83.

38. Bjorner JB, Thunedborg K, Kristensen TS, et al. The Danish SF-36 Health Survey: translation and preliminary validity studies. J Clin Epidemiol 1998;51:991-9.

39. Walmsley RS, Ayres RC, Pounder RE, et al. A simple clinical colitis activity index. Gut 1998;43:29-32.

40. Harvey RF, Bradshaw MJ. Measuring Crohn's disease activity. Lancet 1980;1:1134-5.

41. Lukas C, Landewé R, Sieper J, et al. Development of an ASASEndorsed Disease Activity Score (ASDAS) in patients with ankylosing spondylitis. Ann Rheum Dis 2009;68:18-24.

42. van der Heijde D, Lie E, Kvien TK, et al. ASDAS, a highly discriminatory ASAS-Endorsed Disease Activity Score in patients with ankylosing spondylitis. Ann Rheum Dis 2009;68:1811-8.

43. Machado P, Landewé R, Lie E, et al. Ankylosing Spondylitis Disease Activity Score (ASDAS): defining cut-off values for disease activity states and improvement scores. Ann Rheum Dis 2011;70:47-53.

44. Machado PM, Landewé RB, van der Heijde DM. Endorsement of definitions of disease activity states and improvement scores for the Ankylosing Spondylitis Disease Activity Score: results from OMERACT 10. J Rheumatol 2011;38:1502-6.

45. Christensen R, Bliddal H, Henriksen M. Enhancing the reporting and transparency of rheumatology research: a guide to reporting guidelines. Arthritis Res Ther 2013;15:109.

46. Dougados M, Soubrier M, Perrodeau E, et al. Impact of a nurseled programme on comorbidity management and impact of a patient self-assessment of disease activity on the management of rheumatoid arthritis: results of a prospective, multicentre, randomised, controlled trial (COMEDRA). Ann Rheum Dis 2015;74:1725-33.

47. Peters MJ, Symmons DP, McCarey D, et al. EULAR evidence-based recommendations for cardiovascular risk management in patients with rheumatoid arthritis and other forms of inflammatory arthritis. Ann Rheum Dis 2010;69:325-31.

48. Baillet A, Gossec L, Carmona L, et al. Points to consider for reporting, screening for and preventing selected comorbidities in chronic inflammatory rheumatic diseases in daily practice: a EULAR initiative. Ann Rheum Dis 2016;75:965-73. 